\title{
Uso de Padlet en el Máster Universitario en Didáctica de la Lengua en Educación Infantil y Primaria
}

\author{
Use of Padlet in the University Master's Degree in Language Teaching in Early \\ Childhood and Primary Education
}

\author{
María Aboal López ${ }^{1}$ \\ Universidad Internacional de La Rioja
}

Recibido: 27.07.2021

Aceptado: 30.10 .2021

\section{Resumen}

En la presente propuesta se muestra la puesta en práctica de la pizarra colaborativa Padlet en el aula de la asignatura "La Enseñanza de la Lengua en Contextos Multilingües" del Máster Universitario en Didáctica de la Lengua y la Literatura en Educación Infantil y Primaria de la UNIR. Metodología: A través de una actividad que consiste en diseñar un Padlet para el aula que los estudiantes deben presentar para su Evaluación Continua conseguimos que los futuros docentes se familiaricen con un recurso TIC, de acceso abierto, manejo intuitivo y resultados enriquecedores. Resultados: se presenta aquí el diseño final de la actividad de la pizarra colaborativa propuesta para los alumnos del máster: el enunciado, la manera de evaluarla, así como algunos ejemplos que los estudiantes han realizado en la plataforma en línea de este máster. Conclusiones: se concluye de todo ello que una actividad colaborativa con TIC debe

\footnotetext{
${ }^{1}$ maria.lopezaboal@unir.net https://orcid.org/0000-0002-7236-4167

Volumen 2. Número 1. Enero - Junio 2022 ISSN: 2745-0341 (En línea)
} 
resultar atractiva al usuario, estar especialmente bien presentada, usar recursos intuitivos e incidir, desde el principio, en los resultados de aprendizaje que se esperan.

Palabras clave: Padlet, TIC, trabajo colaborativo, Infantil y Primaria, Didáctica de la Lengua.

\begin{abstract}
This proposal shows the implementation of the collaborative Padlet blackboard in the classroom of the subject "The Teaching of Language in Multilingual Contexts" of the University Master's Degree in Didactics of Language and Literature in Early Childhood and Primary Education of the UNIR. Methodology: Through an activity that consists of designing a Padlet for the classroom that students must present for their Continuous Assessment, we get future teachers to become familiar with an ICT resource, open access, intuitive handling and enriching results. Results: The final design of the collaborative blackboard activity proposed for the master's students is presented here: the statement, the way to evaluate it, as well as some examples that the students have carried out on the online platform of this master's degree. Conclusions: it is concluded from all this that a collaborative activity with ICT must be attractive to the user, be especially well presented, use intuitive resources and influence, from the beginning, the learning results that are expected.
\end{abstract}

Keywords: Padlet, ICT, collaborative work, Infant and Primary, Language Didactics. 


\section{Introducción ${ }^{2}$}

Los trabajos colaborativos resultan necesarios y permiten desarrollar muchas competencias relevantes dentro de cualquier área, si bien en este caso los trabajaremos específicamente en el de lengua. Sin embargo, no siempre los estudiantes se encuentran cómodos ni comprenden las grandes ventajas que este tipo de trabajos les comporta. Al contrario, el alumnado suele centrarse en factores que considera negativos como el de una posible pérdida de tiempo y autonomía que le comporta el tener que trabajar de manera colaborativa con más personas. El problema se agrava, además, si durante el proceso el docente y los discentes tienen que enfrentarse a recursos TIC complejos, para cuyo manejo deben emplear mucha más energía, horas, dedicación y paciencia.

Durante la última década son numerosos los trabajos y artículos sobre el uso de las TIC en el aula de infantil y primaria, si bien luego nos encontramos que la realidad concreta de muchos centros educativos todavía no está realmente inmersa en la nueva era digital. En algunos casos, las diferencias son mayores entre unos países y otros, como hemos podido comprobar con nuestros alumnos del Máster y presentaremos aquí, con grupos diferentes de alumnos de España y de Colombia y Ecuador ${ }^{3}$. No obstante, entre el alumnado español, especialmente el de Primaria (habitualmente más relacionado con el uso en el aula de las nuevas tecnologías), hemos encontrado también discentes no habituados a utilizar este tipo de recursos de la pizarra colaborativa por simple desconocimiento y, en algunos casos incluso, por cierto miedo o respeto al ámbito más tecnológico. Tal y como se viene advirtiendo desde que comenzaron a introducirse las TIC en las aulas de Infantil y Primaria, la integración en el sistema educativo es todavía algo dificultosa especialmente por la escasa formación del profesorado a este

\footnotetext{
${ }^{2}$ Agradecemos y dedicamos este trabajo a todos los estudiantes del curso 2020-21 del Máster en Didáctica de la Lengua y la Literatura en Educación Infantil y Primaria de la Universidad Internacional de La Rioja.

${ }^{3}$ Tal y como advierte Area (2021, p.7): "Uno de los temas relevantes de los últimos años de las agendas de política educativa es la incorporación de las TIC (Tecnologías de la Información y Comunicación) a las escuelas. Este proceso, en los países europeos, comenzó a mediados de los años ochenta (en España, el primer programa institucional se denominó "Proyecto atenea", y en Portugal "minerva") comenzando, en los países latinoamericanos, en la última década del siglo XX como, por ejemplo, la relevante experiencia chilena del Programa enlaces".
} 
respecto (Colás, de Pablos y Ballesta, 2018), por lo que nos parece muy relevante y casi urgente la enseñanza y aprendizaje en este aspecto dentro de cualquier formación para docentes, ya que de ellos depende en gran medida alcanzar una transformación digital real o eficaz en las aulas:

Tanto la educación como la gestión del conocimiento han sufrido una revolución en lo que se refiere a métodos y a herramientas con la entrada en la era digital y el florecimiento y desarrollo de las tecnologías; esta situación ha generado nuevos formatos, conceptos y esquemas de pensamiento que deben saber aplicarse a las distintas asignaturas que integran los programas de estudio que conforman el sistema educativo español en todos sus niveles. Los diferentes currículums educativos nos revelan, efectivamente, la importancia de las TIC y, consecuentemente, en la enseñanza superior se hace necesario incorporar las tecnologías a los programas de estudio de grado y máster de las universidades españolas presenciales o a distancia. (López, 2018, p.32)

Es por estos motivos, y por otros que se irán comentando a lo largo de la presente ponencia, que consideramos de una gran importancia el aprendizaje y uso de herramientas como la pizarra colaborativa Padlet, que permite una manera sencilla y fácil de compartir con los alumnos conocimiento, afianzar el ya adquirido y potenciar la exposición de ideas, diferentes trabajos en sus realidades docentes, etc., otorgando así una amplia perspectiva de cualquier tema que se esté trabajando en el aula.

Lo que se pretende con la actividad planteada que presentamos a continuación es precisamente demostrar a los alumnos del Máster de Lengua en Infantil y Primaria que existen herramientas muy sencillas, útiles y fáciles de manejar que pueden facilitarles mucho el trabajo en el aula, a la vez que les ayuda a conocer otras ideas o proyectos pedagógicos que no conocían y que gracias a las aportaciones comunes del resto de compañeros ellos podrán incluso llegar a utilizar en sus centros educativos. En este sentido, y tal y como nos recuerda Zapata (2016, p.15), dentro de la estrategia pedagógica no podemos considerar exclusivamente el contenido, 
sino que es igual de relevante la manera en la que los alumnos alcanzan o construyen su propio aprendizaje.

Las políticas educativas sobre TIC son necesarias e imprescindibles, pero también son insuficiencias por sí solas. Para que las mismas sean exitosas y tengan impacto real en lo que lo hacen y aprenden los estudiantes en las aulas tienen que ser apropiadas, interiorizadas y reconstruidas personalmente por los agentes educativos. los discursos oficiales son estériles si los mismos no movilizan y estimulan la participación de la sociedad civil que, en nuestro caso, es el profesorado, pero también son otros agentes como los servicios de apoyo externo (inspectores educativos, formadores de docentes, expertos), y la comunidad educativa (familias, agentes sociales, poderes locales). (Area, 2012, p.8)

\section{Metodología}

A través de una actividad que los estudiantes deben presentar para su evaluación continua dentro del Máster Universitario en Didáctica de la Lengua en Educación Infantil y Primaria, podemos comprobar cómo los futuros maestros se familiarizan con un recurso TIC, de acceso abierto, manejo intuitivo y resultados enriquecedores. Los destinatarios de esta propuesta didáctica fueron, en dos convocatorias diferentes, alumnos españoles de un primer grupo (al que a partir de ahora llamaremos grupo A) y alumnos de Hispanoamérica (grupo B que estaba compuesto por alumnos de Colombia y Ecuador). El primero de ellos estaba formado por 48 alumnos que cursaron la asignatura durante el curso 2019/2020 y que trabajaban en diferentes centros educativos españoles. El grupo B estaba compuesto por 27 alumnos que cursaron la asignatura en 2020, y, como en el anterior grupo, también prácticamente todos los alumnos eran docentes en activo y con varios años de experiencia en el aula.

La herramienta escogida para esta actividad TIC es Padlet, una pizarra colaborativa de libre acceso (si bien hay posibilidad de pago según la necesidad del centro educativo). El recurso se encuentra disponible en su propia página web: https://es.padlet.com. Para la implantación 
de este tipo de actividad es conveniente programar previamente una clase o sesión de refuerzo sobre esta actividad, donde podamos enseñar a los alumnos el uso de la pizarra que vamos a explicar a continuación.

Para comenzar a trabajar con esta herramienta previamente debe registrarse el alumno con un correo electrónico (en el caso de que luego esto se traslade a sus alumnos de infantil o primaria lo harán lógicamente con sus tutores legales). Después son varias las opciones de presentación que Padlet permite escoger para crear la primera pizarra colaborativa: Muro, Lienzo, Columna, Lista, Tablero, Cronología, Mapa. Dependiendo del tema que se quiera trabajar lógicamente puede escogerse el tipo de diseño más adecuado, si bien en el caso de nuestra asignatura solemos trabajar con uno de los más sencillos, el del Muro, que permite ir subiendo la información de cada alumno de manera que todas las entradas o aportaciones queden organizadas como diferentes "ladrillos" que facilitan una rápida lectura visual y, en cierta forma, nos acercan a la habitual pizarra de aula o mural grupal.

Al crear el Padlet dentro del diseño escogido nos aparecerá a la derecha una serie de ajustes muy sencillos dentro de los cuales escogeremos el título. En nuestro caso, el de la asignatura o uno similar de su misma temática: "Aula multilingüe" o "Contextos Multilingües en el aula", han sido los escogidos en las dos aulas del Máster que presentamos aquí. Es importante recordar que será también en este lugar donde copiaremos el enlace de esta pizarra en concreto que es el que debemos compartir con el resto del aula para que todos participemos a la vez en la misma pizarra, y evitar problemas o confusiones cuando algunos alumnos crean su propio Padlet, ya que sus intervenciones entonces no aparecerán compartidas con el resto de la clase, perdiendo entonces la actividad su razón de ser (como ya hemos comentando anteriormente estos fueron los casos mínimos pero suponían no superar la actividad).

A partir de la creación del Padlet ya podemos compartir el enlace con los alumnos. Para que se familiaricen lo más rápidamente posible con la nueva herramienta es recomendable en la clase previa a la realización de su actividad mostrarles cómo creamos nosotros mismos la pizarra y escoger incluso el título con ellos. En nuestro caso la enseñanza on line a distancia 
nos permite compartir la pantalla mientras ellos pueden ir siguiendo todos los pasos en sus dispositivos. También en ese momento conviene intervenir nosotros como docentes con un par de propuestas que subiremos en ese momento para que puedan ver lo fácilmente que se agrega cualquier contenido en el muro de nuestra pizarra colaborativa. Y es que, sin duda, este es uno de los principales atractivos de esta herramienta: la facilidad en el uso, ya que simplemente con copiar y pegar en la pizarra colaborativa el enlace de YouTube, Spotify, Instagram o cualquier página de internet o PDF., etc., el enlace o archivo aparece directamente y cualquiera que tenga la dirección de nuestro Padlet puede verlos y abrirlos (es realmente una herramienta muy intuitiva y sencilla de manejar y esto les llama mucho la atención). De este modo, facilitamos a nuestros alumnos el poder participar en una actividad colaborativa útil y atractiva, que desde el inicio les resulta cómoda y sugestiva:

Entendemos por Proyecto Colaborativo con TIC en el contexto educativo, un trabajo en el que intervienen un conjunto de individuos con intereses comunes (alumnos, profesores, padres y comunidad educativa) que participan de forma activa a través de actividades colaborativas, entre equipos de personas y dentro de un proceso de aprendizaje utilizando las Nuevas Tecnologías. (Basilotta y Herrada, 2013, p.3)

\section{Resultados}

A continuación, presentamos el diseño final de la actividad de pizarra colaborativa propuesta para los alumnos del Máster dentro de la asignatura La Enseñanza de la Lengua en Contextos Multilingües: enunciado, rúbrica de evaluación y algunos comentarios que los estudiantes han realizado en la plataforma en línea de este máster.

\section{Pizarra colaborativa multicultural y multilingüe}

A través de la herramienta Padlet, debes aportar al resto de tus compañeros de esta asignatura algún contenido que consideres interesante y fundamental para trabajar por un aula multilingüe y multicultural. Puedes escoger desde leyendas de tu región, contenidos sobre su 
música, sus costumbres y lenguas, y explorar la mejor presentación posible desde las múltiples opciones de esta plataforma (enlaces a canciones o trabalenguas, vídeos, presentaciones, rincones didácticos...).

\section{Objetivos}

. Poner en práctica los conocimientos adquiridos en los temas ya estudiados.

. Buscar información y material en la red de forma autónoma y crítica.

. Crear una actividad a partir de una herramienta TIC.

- Desarrollar el conocimiento para referenciar bibliografía, hemerografía o webgrafía a partir de las normas APA.

. Fomentar el diálogo creativo de ideas entre docentes

\section{Descripción de la actividad}

La profesora os facilitará un enlace de Padlet con la Pizarra Colaborativa de la clase para que puedas participar en aumentar sus contenidos y visualizar las aportaciones del resto de compañeros.

\section{Criterios de evaluación}

. El material escogido es adecuado para su uso en el aula multilingüe.

. La presentación realizada es atractiva y original.

. Todas las fuentes utilizadas están citadas correctamente según las normas APA.

. Se valorará el uso, manejo y aplicación de una herramienta de las nuevas tecnologías.

. Se valorará la forma clara de exponer los materiales, así como su presentación. 


\section{Evaluación}

En la Rúbrica de evaluación el profesor tiene en cuenta los siguientes ítems que comentamos a continuación para valorar las distintas aportaciones al Padlet de sus alumnos. En cada uno de ellos comentamos los aspectos más relevantes para la evaluación de las intervenciones realizadas:

\section{Originalidad.}

Este punto tiene en cuenta si el material compartido en la pizarra colaborativa es original y en qué grado, así como si enriquece de alguna manera lo aprendido en la asignatura y si es adecuado o no al nivel educativo del Master (en nuestro caso Infantil y Primaria).

\section{Perspectiva grupal}

Aquí valoramos si realmente el alumno en sus intervenciones ha mantenido la perspectiva grupal que persigue esta actividad (es decir, no se trata de crear un único Padlet para su aula o sus alumnos, si no participar en el común y hacerlo con ánimo de enriquecer al resto de compañeros). En cierto modo, las aportaciones deben seguir el ritmo de las anteriores, relacionar unas con otras, demostrar el "sentimiento" de grupo, de aula 4

\section{Seguimiento del enunciado}

En este punto se trata de contemplar todo lo que se ha pedido en el enunciado para poder realizar adecuadamente la actividad.

\footnotetext{
${ }^{4}$ En este sentido, Onrubia (2016) nos recuerda que: "lo que hace que la actividad conjunta sea efectivamente conjunta no es la co-presencia física de los participantes, sino el hecho de que profesor y alumnos actúan el uno para el otro y entre sí, de manera que las actuaciones de cada participante sólo se entienden y cobran significado en el marco de, y en referencia a, las actuaciones del resto de participantes".
} 


\section{Exposición de los materiales}

Es fundamental comprobar que la exposición de los distintos materiales presentados por cada discente es adecuada, clara y lo más atractiva posible. Estamos trabajando con un medio muy visual y con esta propuesta pretendemos que practiquen para cuando en su aula hagan un Padlet con o para sus alumnos. Igualmente, aquí se valora la relevancia de los materiales presentados, así como su correcta exposición.

\section{Redacción, formato y citación}

Por último, debemos recordar la importancia de la coherencia y adecuación de cualquier material que no sea original a unas normas de presentación. En los enlaces a vídeos o páginas de internet es más sencillo, si bien cualquier elemento no original (que serán probablemente la mayoría), debe estar correctamente referenciando según, en nuestro caso, la normativa APA. En este mismo apartado valoramos también la redacción del alumno, la adecuación de su texto al ámbito académico y el uso o no de un lenguaje coloquial. Todos los documentos presentados deben hacerlo con formato correcto y con un cuidado uso del lenguaje. Por supuesto, las faltas de ortografía, incluidas las de acentuación, penalizarán la nota final.

En total 37 alumnos del Grupo A (compuesto por 48 alumnos españoles) realizaron esta actividad de Padlet, algo muy sorprendente y positivo cuando 25 de ellos (el 67\%) ya no necesitaban sumar más nota en la evaluación continua, y solo el $32 \%$ por ciento de la clase podía hacerlo. En este se dio el caso de dos archivos no válidos (también podemos hablar de suspenso en cuanto a que no se produce suma final en la nota) como calificación en dicha actividad por no utilizar la pizarra común (objetivo principal) y diseñar un único y exclusivo Padlet sin posibilidad de cooperación del resto de compañeros. Al no seguir estas actividades las exigencias del enunciado ni la perspectiva colaborativa de la actividad en sí, la propuesta en no tenía sentido y no podía calificarse. En el Grupo B de alumnos hispanoamericanos participaron 23 discentes, prácticamente toda la clase, y en este caso había 10 alumnos que sin necesidad de seguir sumando nota también decidieron participar en la actividad 
cooperativa. En este grupo se dio el caso de un archivo no válido, si bien como hemos podido comprobar, en ambos grupos los resultados fueron muy positivos y mejores de lo esperado teniendo en cuenta que se trataba de una actividad nueva que poníamos en marcha en nuestro Máster.

Esta alta participación en la actividad, pese a que muchos sabían que el hacerlo no les aportaría ninguna nota extra para la calificación final, nos demuestra el enorme interés que esta despertó en el alumnado, tanto por la motivación de compartir y conocer así nuevos materiales con el resto de los compañeros como por las ventajas de formarse en una herramienta digital desconocida para el aula. Todo esto manifiesta lo atractivo y útil que los alumnos encontraron esta manera de practicar el aprendizaje cooperativo a través de las TIC, por lo que podemos afirmar que ha sido todo un éxito su implantación en esta asignatura. Por todo ello, hemos ampliado el uso del Padlet a otra asignatura del Grado, como es Didáctica de la Lengua en Educación Infantil, donde hemos sustituido con ella una actividad de foro, ya que conseguimos así mantener el trabajo colaborativo, pero desde una perspectiva más tecnológica y práctica para el día a día del docente en el aula. Los resultados nos han demostrado una mayor satisfacción de los alumnos y un proceso muchísimo más enriquecedor y estimulante de los participantes en el proceso enseñanza/aprendizaje (incluidos, por supuesto, nosotros como docentes):

Los grupos de aprendizaje cooperativo brindan una oportunidad excepcional para hacer un diagnóstico inmediato del aprendizaje de los alumnos, para obtener una retroalimentación inmediata de parte de los pares y para corregir al instante los problemas de comprensión de los estudiantes. (Johnson y Johnson, 1999, p.53)

Por último, cabe recordar que para realizar con éxito la actividad es necesario conocer bien la asignatura, ya que precisamente está ubicada al final de la programación de la misma porque con la misma queríamos conseguir subrayar el carácter integrador de la pizarra colaborativa con los contenidos adquiridos durante todo el curso, pretendiendo así mostrar un análisis de 
los elementos fundamentales que cada alumno quiera destacar de los temas de la asignatura de Contextos Multilingües.

Figura 1. Primer fragmento de la pizarra colaborativa Padlet del Grupo A de la asignatura.

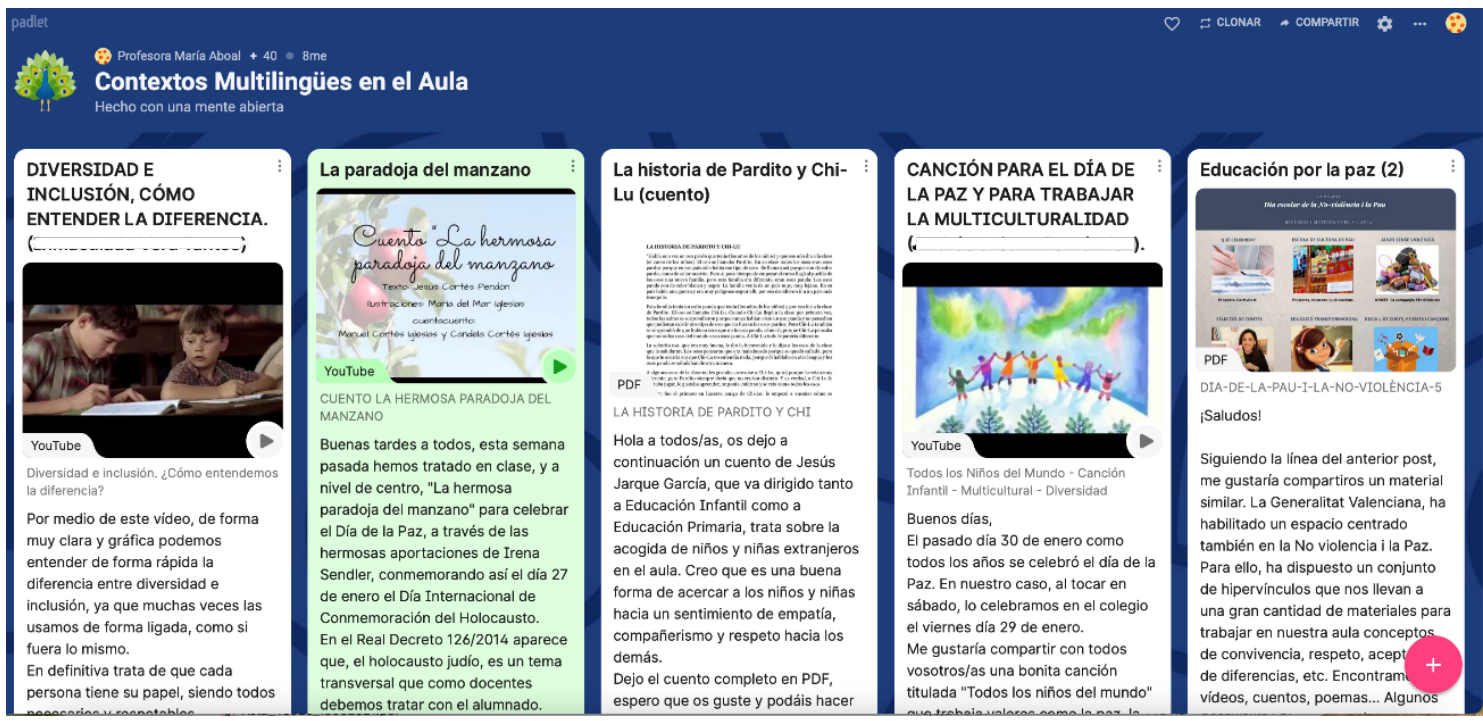

Figura 2. Segundo fragmento de la pizarra colaborativa Padlet del Grupo A de la asignatura.

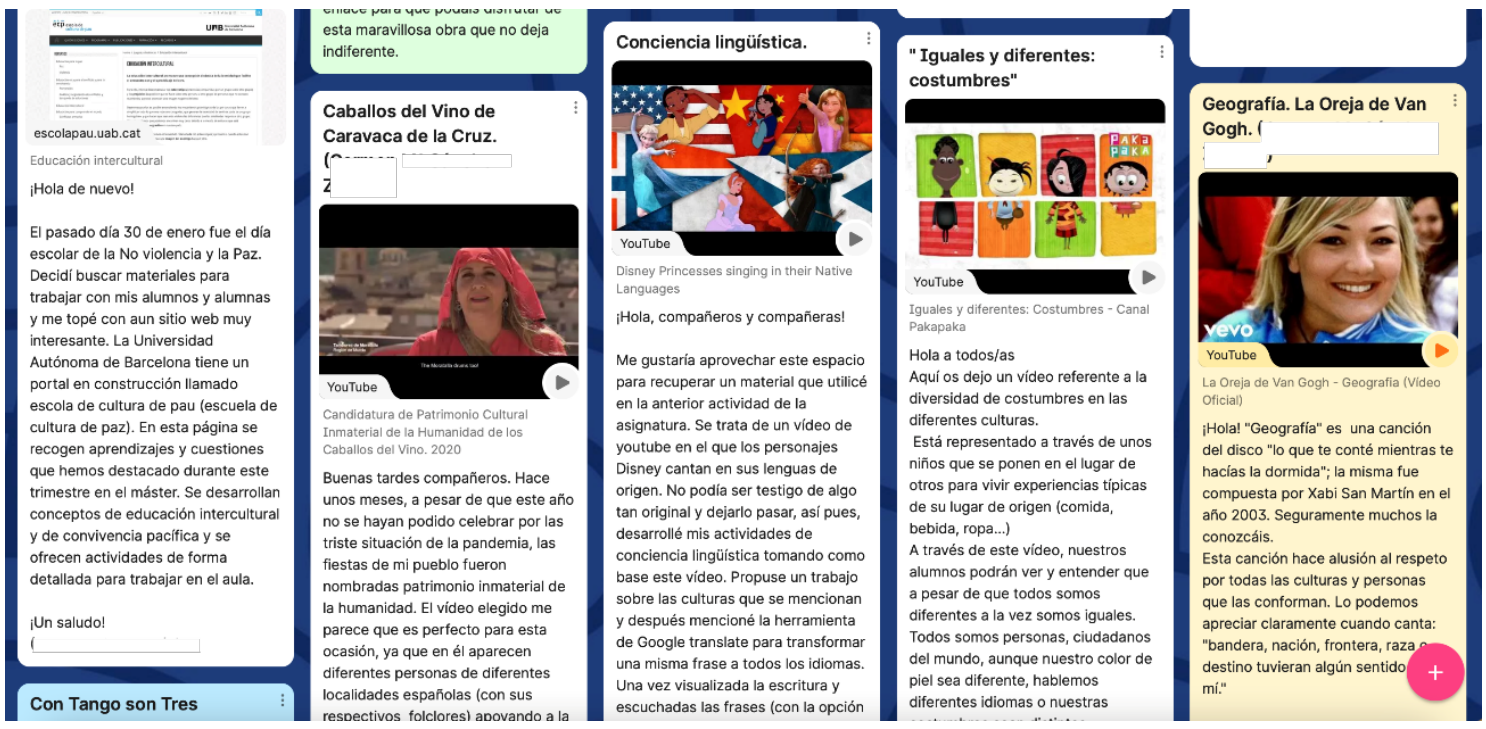


Figura 3. Tercer fragmento de la pizarra colaborativa Padlet del Grupo A de la asignatura.

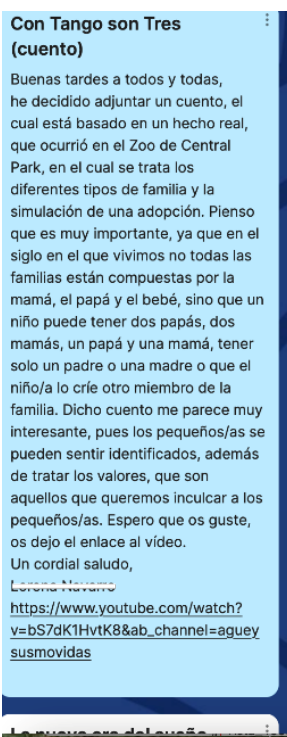

locallaaces espanolas (con sus
respectivos folclores) apoyando a la
decisión de otorgarle este "titulo" a
los Caballos del Vino.
Además, salen subtitulos en inglés
para que otras personas puedan
entender lo que dice el vídeo.
Posible taller multicultural:
"Danzando por el mundo"
(")
Al igual que os he comentado en la
entrada anterior se podrían realizar,
con esa misma estructura o similar,
otros talleres relacionados con otra
vertiente artistica.
2) Taller de danza: En este caso el
objetivo es que el alumnado conozca
diferentes danzas tradicionales de
distintas zonas del mundo. Para ello
se puede realizar una vez a la
semana una pequeña coreografia
inspirada en un baile tradicional de
una parte del mundo.
Por ejemplo: El baile asturianu típico
de Asturias:
https://wwww.youtube.com/watch?
ve Uudkc8v3KSM
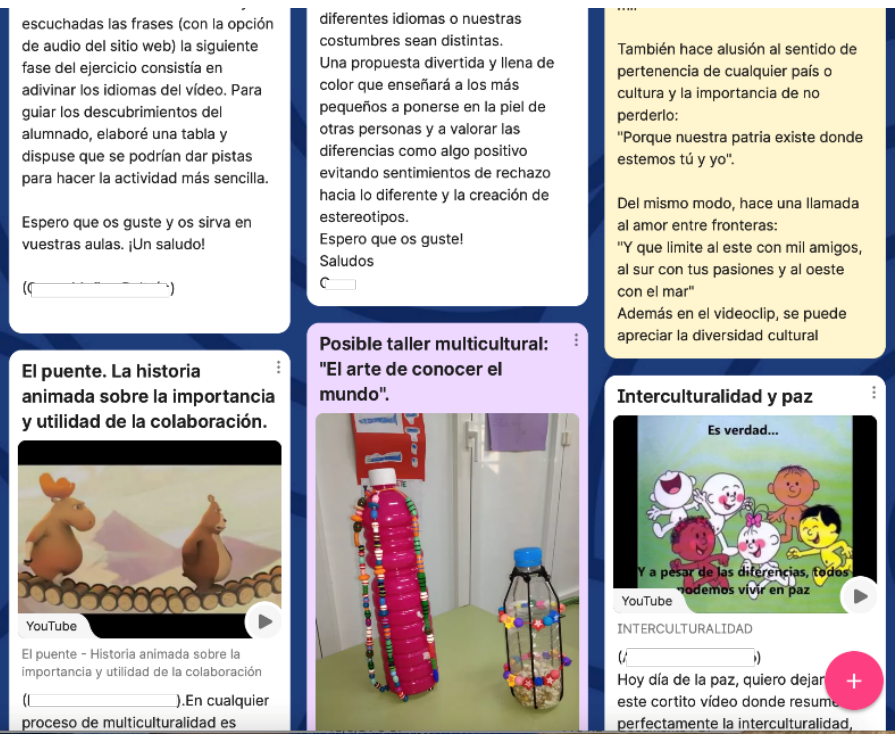

Figura 4. Primer fragmento de la pizarra colaborativa Padlet del Grupo B de la asignatura.
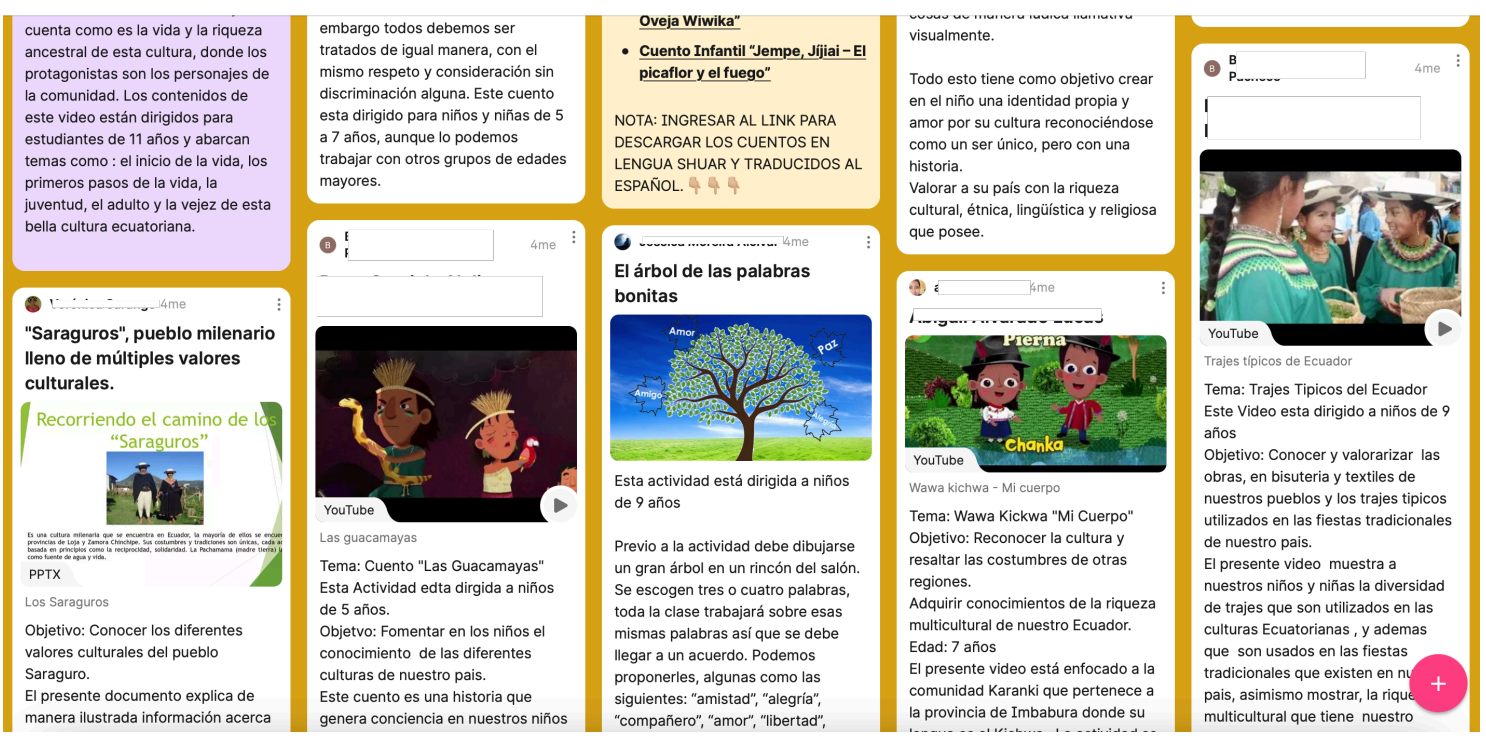
Figura 5. Segundo fragmento de la pizarra colaborativa Padlet del Grupo B de la asignatura.
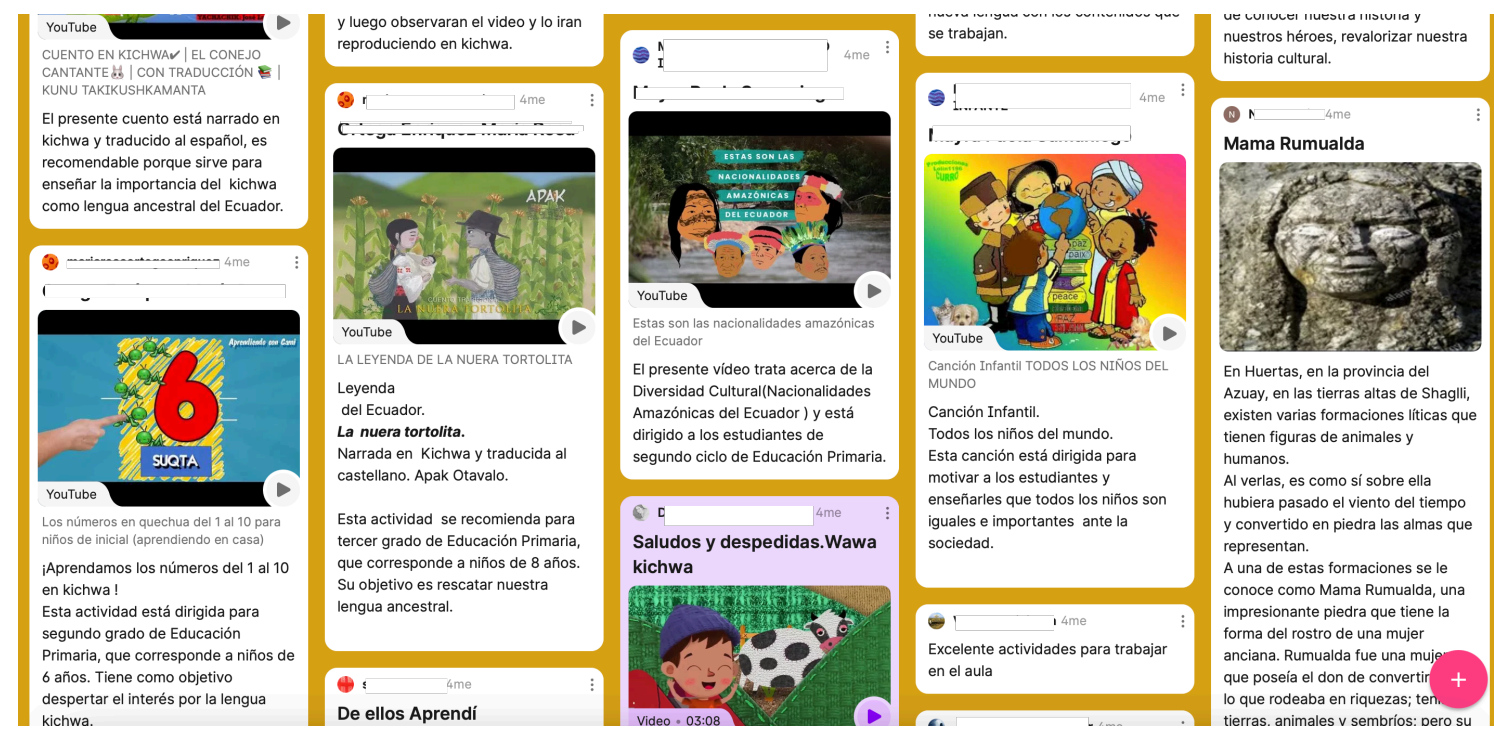

\section{Conclusiones}

Tras la puesta en práctica en el aula de la actividad de Padlet podemos concluir que una actividad colaborativa con TIC debe desde el comienzo resultar atractiva a los alumnos, estar bien presentada, usar recursos intuitivos e incidir, desde el principio, en los resultados de aprendizaje que se esperan. Asimismo, y como hemos podido comprobar con nuestros diferentes grupos, es importante que en la presentación inicial de esta actividad se recalque a los alumnos la necesidad de presentar adecuadamente cualquiera de los recursos, enlaces, vídeos, archivos, etc., que quieran mostrar en esta pizarra común. Es decir, conviene advertir que no debe convertirse en un mero muro de imágenes, canciones o vídeos, sino que una correcta intervención previa explicativa ayuda a encuadrar y entender mejor aquello que se quiere presentar y compartir con el resto del aula. Asimismo, la interacción grupal de unas propuestas y otras dentro de la pizarra colaborativa es lo que hace a la misma cobrar un sentido real de enseñanza/aprendizaje.

Nuestro papel como docentes pasa aquí a una perspectiva constructivista al convertirnos en guías de esta herramienta TIC que ellos deben construir en grupo, de manera coral, como sugerían Johnson y Johnson, a convertirnos en ingenieros que organizan y facilitan el trabajo 
en grupo (Johnson, Johnson y Holubec, 1999, p.4). Esto no significa que desaparezcamos sutilmente de la situación, sino que como defiende Onrubia (2016) desde su perspectiva socioconstructivista, las TIC y los entornos virtuales no reducen ni eliminan nuestro papel como profesores, sino que preciosamente lo amplifican. En este sentido, conviene recordar la afirmación de Onrubia (2016) al analizar el aprendizaje virtual como proceso de construcción:

El aprendizaje virtual, por tanto, no se entiende como una mera traslación o transposición del contenido externo a la mente del alumno, sino como un proceso de (re)construcción personal de ese contenido que se realiza en función, y a partir, de un amplio conjunto de elementos que conforman la estructura cognitiva del aprendiz: capacidades cognitivas básicas, conocimiento específico de dominio, estrategias de aprendizaje, capacidades metacognitivas y de autorregulación, factores afectivos, motivaciones y metas, representaciones mutuas y expectativas (p. 12).

Siguiendo nuevamente a Johnson, Johnson y Holubec (1999, p.4), la herramienta didáctica del aprendizaje cooperativo persigue, como hemos comprobado aquí, elevar el rendimiento de los alumnos y establecer relaciones positivas entre ellos (potenciando además así la diversidad), y la propuesta diseñada para nuestro Máster y presentada aquí pretende explorar y acercarse a las nuevas posibilidades de innovación educativa que nos ofrecen las TIC y con las que debemos seguir experimentando para conseguir una interacción real y eficaz de todos los alumnos en las aulas.

\section{Referencias}

Area, M. (2012). Políticas educativas TIC en los sistemas escolares en Iberoamérica. Miradas desde las dos orillas. Campus $\quad$ Virtuales. 1 (1), 7-9. http://www.uajournals.com/campusvirtuales/jour nal/1/0.pdf

Atúncar-Prieto, C. A. y Medina-Zuta, P. (2021). La virtualización educativa: retos en la formación inicial docente. Maestro y sociedad, 18 (3), 984-1000. 
Basilotta, V. y Herrada, G. (2013). Aprendizaje a través de proyectos colaborativos con TIC. Análisis de dos experiencias en el contexto educativo. EDUTEC, Revista Electrónica de Tecnología Educativa, 44. https://doi.org/10.21556/edutec.2013.44.324.

Cabero, I. y Pallarés, M. (2021). Avances y retos en la implantación de las nuevas tecnologías. En I. Cabero y M. Pallarés (Coords.). Civilización Digital y Pedagogías Emergentes a partir de las Nuevas Tecnologías (pp.13-19). Egregius.

Colás, M., de Pablos, J., y Ballesta, J. (2018). Incidencia de las TIC en la enseñanza en el sistema educativo español: una revisión de la investigación. Revista de Educación aDistancia, 56, 1-56. https://revistas.um.es/red/article/view/321471

Johnson, D., Johnson R. y Holubec, E. (1999). El aprendizaje cooperativo en el aula. Paidós.

Onrubia, J. (2016). Aprender y enseñar en entornos virtuales: actividad conjunta, ayuda pedagógica y construcción del conocimiento. Revista de Educación a Distancia, 50, 1-14. https://doi.org/10.6018/red/50/3.

Zapata, M. (2016). Secuenciación de contenidos y objetos de aprendizaje. Revista de Educación a Distancia, 50, 1-29. http://revistas.um.es/red/article/view/25221/24 521

Zósimo, L. (2018). El diseño de materiales didácticos sobre TIC para una enseñanza universitaria inclusiva y online. Revista Internacional de Comunicación y Desarrollo, 9, 3041. http://dx.doi.org/10.15304/ricd.2.9.5499 